\title{
USP Dissolution Test with Pooled Samples Statistical Analysis of the Third Stage
}

\author{
Nora S. Meneces ${ }^{1}$, Carlos D. Saccone ${ }^{1,2}$ and Julio Tessore1
}

email:csaccone@fing.edu.uy

\begin{abstract}
Dissolution test as defined by USP is an important determination in assessing the quality of different pharmaceutical forms. Some years ago, the pooled samples test was introduced. This test is carried out in three stages like the test for individual samples, but it uses different acceptance criteria. Some doubts have been raised regarding calculation of the third stage average. In this paper, the statistical precision of an equation for this calculation is discussed. Average dissolution values used to assess compliance are affected by several sources of variability: measurement, dissolved amount unit to unit differences, etc. These sources of variability were studied and preferred zones are described for different values of those components, to calculate third stage averages. The results obtained were verified with a simulation study. It was demonstrated that it is possible to reduce the number of units tested in the third stage without introducing a noticeable increase of the risks in the acceptance/rejection decisions.
\end{abstract}

\section{Introduction}

$\mathrm{n}$ the third stage of the USP Dissolution Test with pooled samples (1), (criteria shown in Table 1), the average value of the lot submitted to inspection is compared with the value of the parameter $Q$ specified in the monograph. When the third stage is reached, the calculation of the average of the 3 values obtained in the three stages, can originate the question if the value of the third stage must be weighted double taking in account the fact that they are twelve units instead of six. The following aspects are investigated in this paper:

a. Which is the best estimate of the mean value (weighted vs. non-weighted estimations),

b. Which are the consequences of applying an expression that differs from the optimum for estimation of the mean,

c. Which would be the consequences of taking a sample of only six units also in the third stage instead of twelve.

\section{Discussion}

To perform this work a statistical model of the inspection has been built, including the following sources of variability in the results of each stage:

\begin{tabular}{l}
\hline Table 1. USP Acceptance Table for Pooled Sample \\
\begin{tabular}{|c|c|c|}
\hline Stage & Number units tested & Acceptance Criteria \\
\hline$S 1$ & 6 & $\begin{array}{l}\text { Average amount dissolved is not less than } \\
Q^{*}+10 \%\end{array}$ \\
\hline$S 2$ & 6 & $\begin{array}{l}\text { Average amount dissolved }(S 1+S 2) \text { is } \\
\text { equal to or greater than } Q+5 \% \\
\text { Average amount dissolved }(S 1+S 2+S 3) \text { is } \\
\text { equal to or greater than Q }\end{array}$ \\
\hline $\begin{array}{l}\text { *o is the amount of dissolved active ingredient specified in the individual } \\
\text { monograph, expressed as a percentage of the labeled content. }\end{array}$ \\
\hline
\end{tabular}
\end{tabular}

${ }^{1}$ University of the Republic of Uruguay, Faculty of Engineering, Industrial Production Department, Montevideo, Uruguay
- Product variability: variability between units of the product refers to the dissolved amount in the dissolution test (i.e. variability of the concentration of analyte present in the vessel at the end of the dissolution test). This component was described using the RSD (relative standard deviation). A RSD range of $1-10 \%$ covering the usual values (2) was studied.

- Variability (or uncertainty) due to measurements, including:

- Variability of aliquot volumes used to make the pooled sample. This variability has been modeled using CVp (coefficient of variation of volume delivered); its usual value was considered to be 0.01 (1\%).

- Variability related to the operation of adjusting pooled volume. This variability has been modeled using CVe (coefficient of variation of the filling of the volumetric flask); its usual value was considered to be 0.005 (0.5\%).

- Variability related to the measurement of the concentration of the analyte in the pooled solution. This has been modeled using CVa (coefficient of variation of analysis); figures taken were between 0.005 and 0.03 $(0.5-3 \%)$ in order to consider the different analytical procedures that might be used.

Performing the usual approximation, where: any $C V=\frac{s}{x}$ and any $R S D=\frac{s}{x} .100$, variability of results of each stage is obtained:

$$
C V R_{1}^{2}=\frac{(0.01 R S D)^{2}}{6}+\frac{C V p^{2}}{6}+C V e^{2}+C V a^{2}
$$

${ }^{2}$ Corresponding author, Facultad de Ingeniería, Instituto de Ingeniería Mecánica y Producción Industrial, Julio Herrera y Reissig 565, Montevideo, Uruguay 


\begin{tabular}{|c|c|c|}
\hline \multicolumn{3}{|c|}{$\begin{array}{l}\text { Table2. Analyis of effect on wr } \\
\text { with wariation of RSD and Cwa }\end{array}$} \\
\hline & w value for & wralue for \\
\hline RSD & $\mathrm{CV} / \mathrm{a}=0.005$ & $C V / a=0.03$ \\
\hline 1 & 0,31 & 0,33 \\
\hline 2 & 0,29 & 0,33 \\
\hline 3 & 0,28 & 0,32 \\
\hline 4 & 0,27 & 0,32 \\
\hline 5 & 0,26 & 0,31 \\
\hline $\mathrm{G}$ & 0,26 & 0,31 \\
\hline 7 & 0,26 & 0,30 \\
\hline 8 & 0,26 & 0,30 \\
\hline 9 & 0,25 & 0,29 \\
\hline 10 & 0,25 & 0,29 \\
\hline $\mathrm{CV} / \mathrm{p}=1$ & 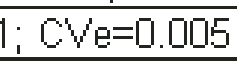 & \\
\hline
\end{tabular}

$C V R_{3}^{2}=\frac{(0.01 R S D)^{2}}{12}+\frac{C V p^{2}}{12}+C V e^{2}+C V a^{2}$

Where:

$R_{1}, R_{2}, R_{3}$ are the pooled results obtained in stage 1,2 and 3 . $C V R_{1}$ is coefficient of variation of result in stage $1, C V R_{2}$ is assumed to be the same as $\mathrm{CVR}_{1}$.

$\mathrm{CVR}_{3}$ is coefficient of variation of result in stage 3 , and RSD is expressed as a percentage.

With the results of stages 1,2 and 3, the mean value of the lot is estimated $(\mathrm{m})$ and the decision of acceptance or rejection is taken by comparing this value with $\mathrm{Q}$.

The method established by USP gives equal weights to the three results despite the fact that they have been obtained with different numbers of dosage units.

In a general way, weighting of the results can be expressed as follows:

$$
m=w R_{1}+w R_{2}+(1-2 w) R_{3}
$$

In this equation the following considerations have been assumed:

- Same weighting is given to results 1 and 2 because they have similar variability,

- Result for stage 3 is weighted by a factor (1-2w), which assures that the three factors add to 1 and $\mathrm{m}$ is an average of the results obtained.

The variability of the calculated mean value $(\mathrm{m})$ is given by:

$$
C V m^{2}=w^{2} C V R_{1}^{2}+w^{2} C V R_{2}^{2}+(1-2 w)^{2} C V R_{3}^{2}
$$

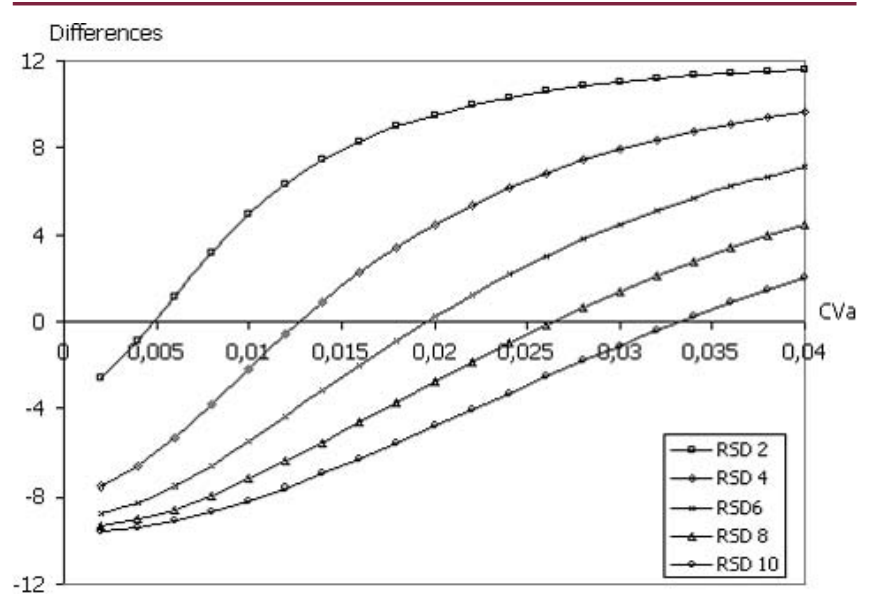

Figure 1. Differences $\left[C V^{2} m(w=1 / 4)-C V 2 m(w=1 / 3)\right] \%$ as a function of CVa for different RSD.

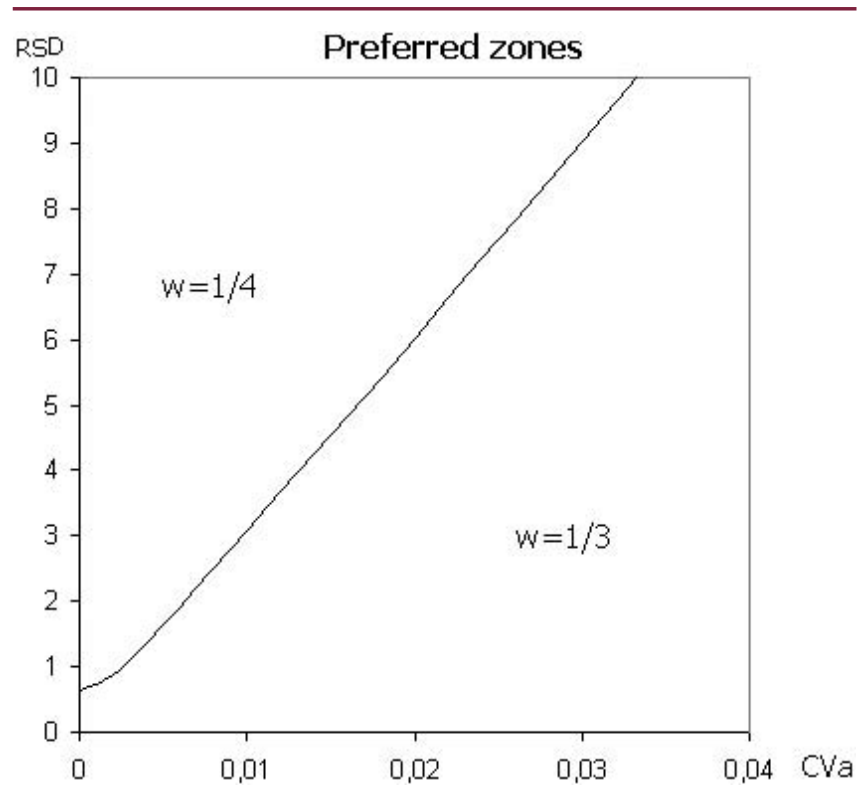

Figure 2. Weighting factors preferred zones (CVp $=0.01$ and $C V e=0.005)$.

The partial derivative of equation 4 with respect to $w$ is:

$$
\frac{\partial C V m^{2}}{\partial w}=4 w C V R_{1}^{2}-4(1-2 w) C V R_{3}^{2}
$$

Then it can be obtained the value of $\mathrm{w}$, which makes minimum the variability of the calculated average value $\mathbf{m}$, and therefore the most efficient estimator of the mean (3). The expression is as follows:

$$
w=\frac{C V R_{3}^{2}}{\left(C V R_{1}^{2}+2 C V R_{3}^{2}\right)}
$$

A value of $w=1 / 3$, indicates that the best estimation is obtained giving to the three results the same weighting, 
TABLE 3- Coefficient of variation of $\mathrm{m}^{*}$

\begin{tabular}{|c|c|c|c|c|c|c|}
\hline \multirow[b]{2}{*}{ RSD } & \multicolumn{3}{|c|}{$C V a=0,005$} & \multicolumn{3}{|c|}{$\mathrm{CV} / \mathrm{a}=0,03$} \\
\hline & $\begin{array}{c}\text { CVm for } \\
\text { w optimum }\end{array}$ & $\begin{array}{c}C V / m \text { for } \\
W=1 / 3\end{array}$ & $\begin{array}{c}C V m \text { for } \\
w=1 / 4\end{array}$ & $\begin{array}{c}\text { CV/m for } \\
\text { w optimum }\end{array}$ & $\begin{array}{c}C V / m \text { for } \\
w=1 / 9\end{array}$ & $w=1 / 4$ \\
\hline 1 & 0,0051 & $0,0 \square 51$ & D, पDE2 & 0,0178 & 0,0178 & 0,0188 \\
\hline 2 & 0, OLE2 & 0,01693 & 0,0063 & 0,0182 & 0,0182 & 0,0192 \\
\hline 3 & 0,0077 & 0,0079 & 0,0070 & 0,0180 & 0,0189 & 0,0197 \\
\hline 4 & 0,0094 & 0,0098 & 0,0095 & 0,0196 & 0,0197 & 0,0204 \\
\hline 5 & 0,0113 & 0,0117 & 0,0113 & 0,0206 & 0,0207 & 0,0213 \\
\hline 6 & 0,0131 & 0,0137 & 0,0131 & 0,0218 & 0,0219 & 0,0224 \\
\hline 7 & 0,0151 & 0,0158 & 0,0151 & 0,0230 & 0,0232 & 0,0236 \\
\hline 8 & 0,0170 & 0,0178 & 0,0170 & 0,0244 & 0,0247 & 0,0249 \\
\hline 9 & 0,0190 & 0,0199 & 0,0190 & 0,0259 & $0, \square 262$ & 0,0262 \\
\hline 10 & 0,0210 & 0,0220 & 0,0210 & 0,0274 & 0,0279 & 0,0277 \\
\hline
\end{tabular}

while a value of $w=1 / 4$ indicates that in order to obtain minimum variability in $\mathbf{m}$ (estimation of mean lot value), double weighting must be given to the stage 3 result.

In order to quantify the results, $w$ has been studied as a function of the analytical parameters and the variability of the lot expressed as RSD. Data are shown in Table 2.

Values obtained show that:

- There is not a unique optimum value of $w$ in the range of parameters considered,

- When the variability of $\mathbf{m}$ is dominated by measurement (low RSD, high $C V a$ ) the optimum $w$ value approaches 1/3, but when product variability dominates (high RSD, low $(\mathrm{V}$ a), optimum value of $w$ approaches $1 / 4$. If measurement variability were negligible, optimum value of $w$ will be $1 / 4$, which means double weighting for the stage 3 value.

To assess the loss of efficiency caused by using a fixed value of $w$, lot average variabilities obtained with an optimum value of $w$ were compared with those obtained using values of $1 / 3$ and $1 / 4$ (data are shown in Table 3 ).

From the analysis of the table it is shown that:

- Variability of measurements is key to determining which weighting is more convenient. A more detailed analysis is shown in Figures 1 and 2.

- The loss of efficiency when $w=1 / 3$ is used (compared with the optimum $w$ value) is not significant; in the worst case, variability increases $4.8 \%$.

Figure 1 shows differences in variability due to weighting factor as a function of RSD (lot variability) and CVa (analysis variability). The difference $\left[\mathrm{CV}^{2} \mathrm{~m}(w=1 / 4)-\mathrm{CV}^{2} \mathrm{~m}(w=1 / 3)\right]$ $\%$ is plotted as a function of $C V a$ for different RSD $(C V p=$ 0.01 and $C V e=0.005)$. Above the $x$-axis, $C V m(w=1 / 4)>$ $\mathrm{CVm}(w=1 / 3)$ and, therefore, $w=1 / 3$ should be preferred. Below the $x$-axis, $w=1 / 4$ is preferred.

Figure 2 shows preferred zones as a function of RSD and $C V a$. In the zone above (below) the curve $w=1 / 4(w=1 / 3)$
Table 4. Coefficient of variation of $m$

\begin{tabular}{|c|c|c|c|c|}
\hline & \multicolumn{2}{c}{ CVa=0,005 } & \multicolumn{2}{c|}{ CV a =0,03 } \\
\hline RSD & $\mathbf{n = 6}$ & $\mathbf{n = 1 2}$ & $\mathbf{n = 6}$ & $\mathbf{n = 1 2}$ \\
\hline $\mathbf{1}$ & 0,0053 & 0,0051 & 0,0179 & 0,0178 \\
\hline $\mathbf{2}$ & 0,0067 & 0,0063 & 0,0183 & 0,0182 \\
\hline $\mathbf{3}$ & 0,0085 & 0,0079 & 0,0191 & 0,0188 \\
\hline $\mathbf{4}$ & 0,0105 & 0,0098 & 0,0201 & 0,0197 \\
\hline $\mathbf{5}$ & 0,0127 & 0,0117 & 0,0213 & 0,0207 \\
\hline $\mathbf{6}$ & 0,0149 & 0,0137 & 0,0227 & 0,0219 \\
\hline $\mathbf{7}$ & 0,0172 & 0,0158 & 0,0242 & 0,0232 \\
\hline $\mathbf{8}$ & 0,0194 & 0,0178 & 0,0259 & 0,0247 \\
\hline $\mathbf{9}$ & 0,0217 & 0,0199 & 0,0276 & 0,0262 \\
\hline 10 & 0,0240 & 0,0220 & 0,0295 & 0,0279 \\
\hline
\end{tabular}

is preferred. This curve was obtained equating variability calculated with $w=1 / 3$ and $w=1 / 4$. Points on the curve have the same variability.

It can be seen in Table 3 that total variability coefficient is a function of RSD and CVa.

Due to the analysis variability contribution, an increase in sample size (6 to 12) reduces variability of the stage 3 average less than could be expected if analysis variability contribution didn't exist.

In order to evaluate the improvement due to additional samples, Table 4 has been prepared. In these calculations, the same values of CVe and $C V p$ were used and a weighting factor of $w=1 / 3$ was applied.

The reduction of variability due to additional samples is less than $9,1 \%$ in all the ranges of parameters studied.

\section{Verification}

In order to verify the theoretical analysis, simulations were performed taking into account the statistical model outlined.

Each value shown in Table 5 is the average of 1000 repetitions and the resultant confidence interval (95\%) is approximately 0.06 .

The results of the simulation are in agreement with cal- 
Table 5: Theoretical vs "Experimental" Variation Coefficientes of $m$

\begin{tabular}{|c|c|c|c|c|c|c|c|c|}
\hline & \multicolumn{4}{|c|}{$C V a=0,005$} & \multicolumn{4}{|c|}{$C V a=0,03$} \\
\hline & \multicolumn{2}{|c|}{ Theoretical } & \multicolumn{2}{|c|}{ Simulation } & \multicolumn{2}{|c|}{ Theoretical } & \multicolumn{2}{|c|}{ Simulation } \\
\hline RSD & $a=1 / 3$ & $a=1 / 4$ & $\mathrm{a}=1 / 3$ & $a=1 / 4$ & $a=1 / 3$ & $a=1 / 4$ & $a=1 / 3$ & $a=1 / 4$ \\
\hline$\overline{1}$ & 0,0051 & 0,00 & 0,0049 & 0,0050 & 0178 & 0,0188 & 0178 & 0193 \\
\hline 2 & 0,0063 & 0,0063 & 0,0066 & 0,0067 & 0,0182 & 0,0192 & 0,0185 & 0,0198 \\
\hline$\overline{3}$ & 0,0079 & 0,0078 & 0,0081 & 0,0078 & 0,0188 & 0,0197 & 0,0185 & 0,0195 \\
\hline 4 & 0,0098 & $0,0 !$ & 0,0095 & 0,0 & 0,0197 & 0,020 & 0196 & 0,0204 \\
\hline$\overline{5}$ & 0,0117 & 0,0113 & 0,0118 & 0,0113 & 0,0207 & 0,0213 & 0,0203 & 0,0214 \\
\hline 6 & 0,0137 & 0,0131 & 0,0141 & 0,0133 & 0,0219 & 0,0224 & 0,0215 & 0,0220 \\
\hline 7 & 0,0158 & 0,0151 & 0,0161 & 0,01 & 0,0232 & 0,0236 & 0,0239 & 0,0242 \\
\hline 8 & 0,0178 & 0,0170 & 0,0170 & 0,0 & 0,0247 & 0,0249 & 0,0243 & 0,0246 \\
\hline$\overline{9}$ & 0,0199 & 0,0190 & 0,0205 & 0,0195 & 0,0262 & 0,0262 & 0,0255 & 0,0255 \\
\hline 10 & 0,0220 & 0,0210 & 0,0218 & 0,0208 & 0,0279 & 0,0277 & 0,0268 & 0,0266 \\
\hline
\end{tabular}

culations within the confidence intervals. The maximum observed difference $(a=1 / 4, \mathrm{RSD}=2$ and $C v a=0.005)$ is $5.9 \%$.

\section{Conclusions}

The optimum weighting factor is variable. Depending on the relative values of the sources of variability, equal weighting (as established by USP 27) or double weighting third stage, provides estimators of lot mean with less variability. In any case, loss of efficiency (increase in variability) due to the use of a fixed, equal weighting to calculate average is not significant. Third stage testing could be reduced to six units without introducing a noticeable increase of risks in the acceptance/rejection decisions since there is little increase in the variability of the estimated average.

\section{Acknowledgement}

The authors wish to thank Ms. Elena Saccone for her language revisions of the manuscript.

\section{References}

1. THE UNITED STATED PHARMACOPOEIA 27, The U.S.

Pharmacopoeia Convention, Inc., Board of Trustees, Webcom Limited, Toronto, Ontario, 2303-2304, 2004.

2. Hofer, Jeffrey D., Gray Vivian A, "Examination of Selection of Immediate Release Dissolution Acceptance Criteria", Dissolution Technologies, 10 (1, February), 16-20, 2003.

3. Montgomery, D.,"Introduction to statistical quality control', th $^{\text {th }}$ ed., John Wiley \& Sons, Inc., New York, NY, 91, 2001. 